\title{
Hsa-let-7c controls the committed differentiation of IGF-1-treated mesenchymal stem cells derived from dental pulps by targeting IGF-1R via the MAPK pathways
}

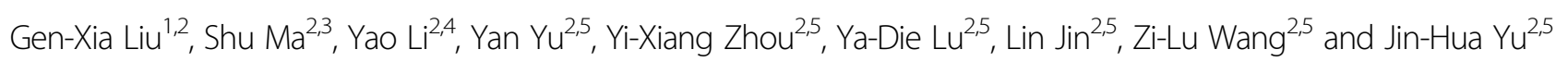

\begin{abstract}
The putative tumor suppressor microRNA let-7c is extensively associated with the biological properties of cancer cells. However, the potential involvement of let- $7 c$ in the differentiation of mesenchymal stem cells has not been fully explored. In this study, we investigated the influence of hsa-let-7c (let-7c) on the proliferation and differentiation of human dental pulp-derived mesenchymal stem cells (DPMSCs) treated with insulin-like growth factor 1 (IGF-1) via flow cytometry, CCK-8 assays, alizarin red staining, real-time RT-PCR, and western blotting. In general, the proliferative capabilities and cell viability of DPMSCs were not significantly affected by the overexpression or deletion of let-7c. However, overexpression of let-7C significantly inhibited the expression of IGF-1 receptor (IGF-1R) and downregulated the osteo/odontogenic differentiation of DPMSCs, as indicated by decreased levels of several osteo/odontogenic markers (osteocalcin, osterix, runt-related transcription factor 2, dentin sialophosphoprotein, dentin sialoprotein, alkaline phosphatase, type 1 collagen, and dentin matrix protein 1) in IGF-1-treated DPMSCs. Inversely, deletion of let$7 c$ resulted in increased IGF-1R levels and enhanced osteo/odontogenic differentiation. Furthermore, the ERK, JNK, and P38 MAPK pathways were significantly inhibited following the overexpression of let-7c in DPMSCs. Deletion of let-7c promoted the activation of the JNK and P38 MAPK pathways. Our cumulative findings indicate that Let-7C can inhibit the osteo/odontogenic differentiation of IGF-1-treated DPMSCs by targeting IGF-1R via the JNK/P38 MAPK signaling pathways.
\end{abstract}

\section{Introduction}

Insulin-like growth factor 1 (IGF-1) is a polypeptide hormone that is mainly synthesized by the liver. It is the most abundant growth factor in the bone matrix and

\footnotetext{
Correspondence: J-H. Yu (yujinhua@njmu.edu.cn)

${ }^{1}$ Endodontic Department, Hefei Hospital of Stomatology, 265 Changjiang

Middle Road, 230001 Hefei, Anhui, China

${ }^{2}$ Key Laboratory of Oral Diseases of Jiangsu Province and Stomatological

Institute of Nanjing Medical University, 136 Hanzhong Road, 210029 Nanjing, Jiangsu, China

Full list of author information is available at the end of the article

These authors contributed equally: Gen-Xia Liu, Shu Ma.
}

plays an important role in bone maintenance and remodeling $^{1-3}$. In general, IGF-1 mediates cell behaviors via the IGF-1 receptor (IGF-1R, a transmembrane tyrosine kinase-containing receptor). Following binding of the IGF-1 ligand, IGF-1R can activate downstream signaling pathways that subsequently regulate cell growth, apoptosis, mineralization, differentiation, and osteogenesis ${ }^{4}$, and thus the IGF-1/IGF-1R pathway is known as the IGF-1/IGF-1R axis ${ }^{5-8}$.

Various studies have demonstrated that IGF-1 can promote the proliferation and osteo/odontogenic

\section{(c) The Author(s) 2018}

\footnotetext{
(c) (i) (-) O) Open Access This article is licensed under a Creative Commons Attribution-NonCommercial-NoDerivatives 4.0 International License, which permits any non-commercial cc) ${ }_{\text {BY NC ND }}$ use, sharing, distribution and reproduction in any medium or format, as long as you give appropriate credit to the original author(s) and the source, and provide a link to the Creative Commons license. You do not have permission under this license to share adapted material derived from this article or parts of it. The images or other third party material in this article are included in the article's Creative Commons license, unless indicated otherwise in a credit line to the material. If material is not included in the article's Creative Commons license and your intended use is not permitted by statutory regulation or exceeds the permitted use, you will need to obtain permission directly from the copyright holder. To view a copy of this license, http:// creativecommons.org/licenses/by-nc-nd/4.0/
} 
differentiation of mesenchymal stem cells (MSCs) in different tissues ${ }^{9}$. Endogenous and exogenous IGF-1 signaling mediated through IGF-IR plays an important role in the differentiation and morphogenesis of human embryonic stem cells in three-dimensional culture ${ }^{10}$. IGF1 can significantly improve the proliferative and survival capabilities of neural progenitor-like cells derived from bone marrow mesenchymal stem cells (BMSCs) ${ }^{9}$. Moreover, low IGF-1 has been shown to be a risk factor for osteoporosis and bone fractures ${ }^{11}$. IGF-1/IGF-1R signaling has also been shown to be important for both the terminal differentiation of osteoprogenitors from bone MSCs and bone mass acquisition ${ }^{3}$. Thus, the IGF-1/IGF$1 \mathrm{R}$ axis plays a critical role in the proliferation and osteogenic differentiation of MSCs. IGF-I and insulin activate ERK1/2 mitogen-activated protein kinase (MAPK) via the type 1 IGF receptor in mouse embryonic stem cells ${ }^{12}$. Our previous studies have demonstrated that IGF-1 can upregulate the osteo/odontogenic differentiation of MSCs by activating the MAPK signaling pathways $^{13-15}$. During the process of bone resorption, the release of IGF-1 from the bone matrix can induce the differentiation of MSCs toward an osteoblast lineage by activating mTOR signaling to maintain the bone microarchitecture and mass ${ }^{2}$. Moreover, IGF/IGF-1R can indirectly stimulate the PI3K/Akt pathway in an interactive osteogenic signaling network, which is necessary for both bone development and remodeling ${ }^{16}$. Despite the enormous progress in the mechanistic, pathway-level understanding of IGF-1-mediated differentiation of MSCs, there is still a lack of understanding of the committed differentiation induced by IGF-1 at the microRNA (miRNA) level.

miRNAs are non-coding RNAs that are 21-23 nucleotides in length and posttranscriptionally regulate protein expression by directly binding to the $3^{\prime}$-untranslated regions ( $3^{\prime}$-UTRs) of target genes. They play important roles in numerous biological processes, including development, apoptosis, longevity, and metabolism ${ }^{17,18}$. Originally discovered in the nematode Caenorhabditis elegans, let-7 miRNA plays a critical role in regulating cell proliferation and differentiation and also participates in the maintenance of stem cell niches ${ }^{19,20}$. Let-7c is one member of the let-7 family; it maps to human chromosome 21q11-21 and is known as a putative tumor suppressor in several cancer cell lines ${ }^{21-23}$. Further, let-7c markedly promotes ectopic bone formation and suppresses adipogenesis by targeting the high-mobility group AT-hook 2 in MSCs derived from human adipose tissues $^{24}$. Differentiation of MSCs is usually under precise regulation by multiple modifiers, including miRNAs and related signaling pathways ${ }^{25-27}$. To date, little is known about the role of let- $7 c$ and its involvement in pathways that are critical for the committed differentiation of IGF1-treated MSCs.

In the present study, we investigated the potential gene targets of $h s a-l e t-7 c$ by TargetScan, miRDB, and microRNA.org; clarified the interaction between let-7c and IGF-1R as well as the effects of these two genes on the proliferation and differentiation of dental pulp MSCs; and further explored the potential involvement of let-7c in various signaling pathways.

\section{Materials and methods \\ Cell isolation and culture}

Normal human impacted third molars were collected with informed consent from patients (18-25 years of age) in the Oral Surgery Department of Jiangsu Provincial Stomatological Hospital. Dental pulp was carefully isolated from the impacted third molars and primary DPMSCs were enzymatically separated, as reported previously $^{28-30}$. These cells were purified using rabbit antiSTRO-1 antibody (Santa Cruz, Delaware, CA) and sheep anti-rabbit IgG Dynabeads (Dynal Biotech, Oslo, Norway) according to the standard procedure for magneticactivated cell sorting. Purified DPMSCs were seeded at $1 \times 10^{4}$ cells $/ \mathrm{mL}$ into $6 \mathrm{~cm}$ culture dishes and subsequently cultured in alpha minimum essential medium $(\alpha-$ MEM, Gibco, Life Technologies, Grand Island, NY, http:// www.lifetechnology.com/) supplemented with $10 \%$ fetal bovine serum (FBS, Gibco, Life Technologies, USA), 100 $\mu \mathrm{g} / \mathrm{mL}$ streptomycin, and $100 \mathrm{U} / \mathrm{mL}$ penicillin at $37^{\circ} \mathrm{C}$ in a humidified, $5 \% \mathrm{CO}_{2}$ atmosphere. Cells were subcultured at a $1: 3$ ratio when they reached $80-90 \%$ confluence and the culture medium was replaced every 2 days. DPMSCs passaged between three and five times were used in the following experiments.

\section{Cell identification}

To investigate the origin of isolated multiclonal cells, cells were harvested and incubated with various combinations of the following fluorochrome-conjugated rabbit anti-human antibodies (CD34-FITC, CD146-APC, CD105-APC, CD73-PE, CD45-PerCP, CD90-PE; Miltenyi, Germany, http://www.miltenyibiotec.com) and rat antihuman antibody STRO-1 (BioLegend, USA, http://www. biolegend.com) for $20 \mathrm{~min}$ at room temperature in the dark. The corresponding mouse IgG isotype control antibodies conjugated to FITC, APC, PE, or PerCP were employed as negative controls in each experiment. 0.01 $\mathrm{mol} / \mathrm{L}$ PBS was used as a negative control for STRO-1 staining. Stained cells were washed twice with $0.01 \mathrm{~mol} / \mathrm{L}$ PBS and analyzed by flow cytometry (FCM) (FACSCalibur, BD Biosciences, USA). 


\section{Lentivirus transfection}

LV-hsa-let-7c and LV-hsa-let-7c-inhibition lentiviruses were purchased from GENECHEM (Shanghai, China, http://www.genechem.com.cn/). The LV-hsa-let-7c $(+$ Let-7c) vector consisted of the pre-hsa-let-7c sequence cloned into a GV217 plasmid. A GV217 plasmid expressing a scrambled RNA was used as a control (+Ctrl). The LV-hsa-let-7c-inhibition $(-$ Let-7c) vector consisted of the reverse complement of the mature hsalet-7c transcript cloned into a GV159 plasmid. A GV159 plasmid expressing a scrambled RNA was used as a control $(-\mathrm{Ctrl})$. For better observation, all lentiviruses encoded a green fluorescence protein (GFP) gene sequence. DPMSCs at $60-70 \%$ confluence were transfected with let-7c lentiviruses for $12 \mathrm{~h}$ in $2 \mathrm{~mL} \alpha$-MEM supplemented with $10 \% \mathrm{FBS}$ and $0.05 \mathrm{mg} / \mathrm{mL}$ polybrene (Santa Cruz, USA).

\section{Cell counting kit-8 assay}

DPMSC proliferation was measured using the commercial cell counting kit (CCK)-8 in accordance with the manufacturer's protocol. Briefly, cells were seeded into 96-well cell culture cluster plates (Corning, Corning, NY, USA) at an initial density of $3 \times 10^{3}$ cells/well in $\alpha$-MEM containing 10\% FBS until $60 \%$ confluence, and then serum-starved for $24 \mathrm{~h}$.

CCK-8 reagents (Dojindo, Kumamoto, Japan, http:// www.dojindo.eu.com) were added to a subset of wells at the indicated time points, and the absorbance was quantified using an automated plate reader. Absorbency was measured at a test wavelength of $490 \mathrm{~nm}$ and a reference wavelength of $650 \mathrm{~nm}$ using a micro-plate reader (Bio$\mathrm{Rad})$. CCK-8 results were expressed as the mean \pm standard deviation (SD) and the experiment was performed three times.

\section{Flow cytometry}

Cells in each group were harvested with trypsin (Beyotime, Haimen, China) and fixed with $75 \%$ ice-cold ethanol at $4{ }^{\circ} \mathrm{C}$ for $24 \mathrm{~h}$ in the dark. Then, each sample was measured using a FAC-Scan flow cytometer (BD Biosciences, San Jose, CA). Cell cycle fractions (G0/G1, S, and $\mathrm{G} 2 / \mathrm{M}$ phases) and cell apoptosis levels were determined by FCM. Three independent experiments were performed for each group.

\section{Alizarin red staining}

To evaluate the osteogenic differentiation of + Let-7cl -Let-7c DPMSCs, DPMSCs were seeded into 24-well plates (Corning) at a density of $1 \times 10^{4}$ cells/well, infected with + Let- $7 c /-$ Let- $7 c$ lentiviruses and then incubated in four different media $(\alpha-M E M, \alpha-M E M+100 \mathrm{ng} / \mathrm{mL}$ IGF1, MM, MM+100 ng/mL IGF-1). MM is the mineralization-inducing medium containing $\alpha$-MEM,
$10 \% \mathrm{FBS}, 100 \mathrm{U} / \mathrm{mL}$ penicillin, $100 \mu \mathrm{g} / \mathrm{mL}$ streptomycin, $2 \mathrm{mmol} / \mathrm{L}$ L-glutamine (Sigma-Aldrich, American, http:// www.sigmaaldrich.com/), $50 \mathrm{mg} / \mathrm{L}$ ascorbic acid (SigmaAldrich, American), $10 \mathrm{mmol} / \mathrm{L} \quad \beta$-glycerophosphate (Sigma-Aldrich, American), and $10 \mathrm{nmol} / \mathrm{L}$ dexamethasone (Sigma-Aldrich, American).

After 2 weeks of mineralization induction, cells were fixed in ice-cold $70 \%$ ethanol for $30 \mathrm{~min}$ and stained with alizarin red ( $40 \mathrm{mM}, \mathrm{pH}=4.2$, Sigma-Aldrich) for $5 \mathrm{~min}$ at room temperature. Images were acquired using a scanner and alizarin red was destained with $10 \%$ cetylpyridinium chloride $(\mathrm{CPC})$ in $10 \mathrm{mmol} / \mathrm{L}$ sodium phosphate for $30 \mathrm{~min}$ at $25^{\circ} \mathrm{C}$. Calcium concentrations were determined according to the absorbance reading at 562 $\mathrm{nm}$ using a standard calcium curve prepared in the same solution. The final calcium levels in each group were normalized with the total protein concentrations obtained from the duplicate plates.

\section{Real-time reverse transcription polymerase chain reaction (real-time RT-PCR)}

DPMSCs were cultured in $6 \mathrm{~cm}$ dishes until they reached $60-70 \%$ confluence. Then, they were transfected with let-7c lentiviruses for $12 \mathrm{~h}$ in $2 \mathrm{~mL} \alpha$-MEM supplemented with $10 \% \mathrm{FBS}$ and $0.05 \mathrm{mg} / \mathrm{mL}$ polybrene. After $12 \mathrm{~h}$ of transfection, DPMSCs were cultured in $3 \mathrm{~mL} \alpha$ MEM supplemented with $10 \% \mathrm{FBS}, 100 \mu \mathrm{g} / \mathrm{mL}$ streptomycin, $100 \mathrm{U} / \mathrm{mL}$ penicillin, and $100 \mathrm{ng} / \mathrm{mL}$ IGF- 1 for 0 , 3 , and 7 days before they were harvested for RT-PCR. Total cellular RNA was extracted from cells in each group using TRIzol reagent (Invitrogen, Carlsbad, USA, https:// www.thermofisher.com) according to the manufacturer's instructions. Isolated RNA precipitates were completely dissolved in diethylpyrocarbonate-treated water (Ambion Inc., Austin, USA) and reverse transcribed using a PrimeScript RT Master Mix kit (TaKaRa, Dalian, China). For the analysis of let-7c over/underexpression, isolated RNA was subjected to reverse transcription with SMART ${ }^{\circ}$ MMLV Reverse Transcriptase (Takara Bio, Otsu, Japan). Real-time RT-PCR was performed using the SYBR ${ }^{\infty}$ Premix Ex Taq ${ }^{\text {Tit }}$ kit (TaKara Bio, Otsu, Japan) on an ABI 7300 real-time PCR system. The mRNA expression levels of hsa-let-7c and several osteo/odontogenic markers, including osteocalcin $(O C N)$, osterix $(O S X)$, runt-related transcription factor 2 (RUNX2), dentin sialophosphoprotein $(D S P P)$, alkaline phosphatase $(A L P)$, collagen type $1(C O L-1)$, and dentin matrix protein-1 (DMP-1), were detected using the primer sets listed in Table 1 . Realtime RT-PCR reaction conditions were: $95^{\circ} \mathrm{C}$ for $30 \mathrm{~s}$, followed by 40 cycles of $95^{\circ} \mathrm{C}$ for $5 \mathrm{~s}$ and $60^{\circ} \mathrm{C}$ for $31 \mathrm{~s}$. GAPDH (glyceraldehyde-3-phosphate dehydrogenase) was employed as the reference housekeeping gene for normalizing mRNA levels of osteo/odontogenic genes. U6 was employed as the reference housekeeping gene for 
Table 1 Sense and antisense primers for real-time RT-PCR

\begin{tabular}{|c|c|c|c|}
\hline Gene & GenBank No. & Sequences $\left(5^{\prime}-3^{\prime}\right)$ & Size (bp) \\
\hline \multirow[t]{2}{*}{ OCN } & NM_001199662.1 & Forward: AGCAAAGGTGCAGCCTITGT & 63 \\
\hline & & Reverse: GCGCCTGGGTCTCTTCACT & \\
\hline \multirow[t]{2}{*}{ os $X$} & NM_152860.1 & Forward: CTCCTGCGACTGCCCTAA & 125 \\
\hline & & Reverse : GCGAAGCCTTGCCATACA & \\
\hline \multirow[t]{2}{*}{ DSPP } & NM_014208.3 & Forward: ATATTGAGGGCTGGAATGGGGA & 136 \\
\hline & & Reverse: TTTGTGGCTCCAGCATTGTCA & \\
\hline \multirow[t]{2}{*}{ RUNX2 } & NM_001024630.3 & Forward: TCTTAGAACAAATTCTGCCCTTT & 136 \\
\hline & & Reverse: TGCTTTGGTCTTGAAATCACA & \\
\hline \multirow[t]{2}{*}{$A L P$} & NM_000478.5 & Forward: GACCTCCTCGGAAGACACTC & 137 \\
\hline & & Reverse: TGAAGGGCTTCTTGTCTGTG & \\
\hline \multirow[t]{2}{*}{ COL-1 } & NM_000090.3 & Forward: GAGCTGGCTACTTCTCGC & 103 \\
\hline & & Reverse: TCTATCCGCATAGGACTGAC & \\
\hline \multirow[t]{2}{*}{ DMP1 } & NM_004407.3 & Forward: TITAAGGAAGTCTCGCATCT & 209 \\
\hline & & Reverse: TGGGACCATCTACGTITT & \\
\hline \multirow[t]{2}{*}{ GAPDH } & NM_002046.3 & Forward: GAAGGTGAAGGTCGGAGTC & 225 \\
\hline & & Reverse: GAGATGGTGATGGGATTTC & \\
\hline
\end{tabular}

analysis of $h s a-l e t-7 c$ levels. The results were calculated from three independent experiments. Relative gene expression values were calculated by the $2^{-\Delta \Delta \mathrm{Ct}}$ method, as previously described ${ }^{31,32}$. All PCR reactions were performed in triplicate and data are expressed as the mean \pm SD. Primers used for the detection of hsa-let-7c are as follows: U6, forward 5'-CTCGCTTCGGCAGCACA-3' and reverse $5^{\prime}$-AACGCTTCACGAATTTGCGT-3', hsa-let-7c, forward 5' -ACACTCCAGCTGGGTGAGGTA GTAGGTTGT-3' and reverse 5'-TGGTGTCGTGGAGT CG-3'.

\section{Western blot}

Cells used for Western blot analysis were cultured identically to those used for RT-PCR and were harvested at days 0,3 , and 7 for the following experiments. After washing twice with cold PBS, cells were lysed for $15 \mathrm{~min}$ on ice in RIPA lysis buffer (Beyotime, China) supplemented with $1 \mathrm{mM}$ phenylmethylsulfonyl fluoride (Beyotime). Cell debris was eliminated by centrifugation at $12,000 \mathrm{rpm}$ for $10 \mathrm{~min}$. Protein concentrations were determined using the Bio-Rad protein assay kit (Pierce, Rockford, IL). $20 \mu \mathrm{g}$ of each protein sample was used for Western blot analysis according to the previously described protocol. For the analysis of signaling pathways, cytoplasmic and nuclear proteins were extracted with NEPER $^{\oplus}$ Nuclear and Cytoplasmic Extraction Reagents (Thermo Scientific, USA), respectively, at the indicated time points and subsequently analyzed by Western blot.
The following primary antibodies were used: IGF-1R (1:200, BOSTER, http://boster.com.cn), OCN (1:1000, Abcam, http://www.abcam.com/), OSX (1:1000, Abcam), RUNX2 (1:1000, Bioworld, http://www.bioworld.com/), DSP (1:1000, Santa Cruz), ALP (1:1000, Abcam), COL-1 (1:1000, Abcam), DMP-1 (1:1000, Abcam), ERK1/2 (1:1000, Bioworld), phosphor-ERK1/2 (1:1000, Bioworld), JNK1/2/3 (1:1000, Bioworld), phosphor-JNK1/2/3 (1:1000, Bioworld), P38 (1:1000, Bioworld), phosphor-P38 (1:1000, Bioworld), and $\beta$-ACTIN (1:1000, Bioworld). Experiments were performed in triplicate using $\beta$-ACTIN as the internal control.

\section{Statistical analysis}

Statistical analysis was performed using SPSS software v17.0. Quantitative results are expressed as the mean \pm SD. Data were analyzed by a paired Student's $t$-test (twotailed distribution). $P$-values less than 0.05 were regarded as statistically significant.

\section{Results}

Let-7c inhibits IGF-1R expression in DPMSCs

In culture, DPMSCs exhibited spindle-like and fibroblast-like morphology (Fig. 1a, b). FCM analysis showed that DPMSCs stained positively for several MSC markers, including STRO-1, CD73, CD90, CD105, and CD146, but stained negatively for the hematopoietic markers CD34 and CD45 (Fig. 1c, d). 
a

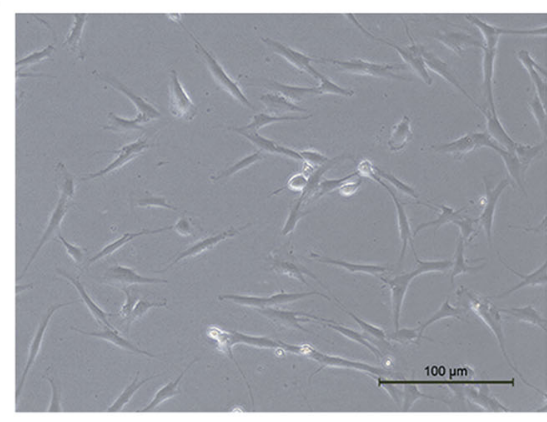

b

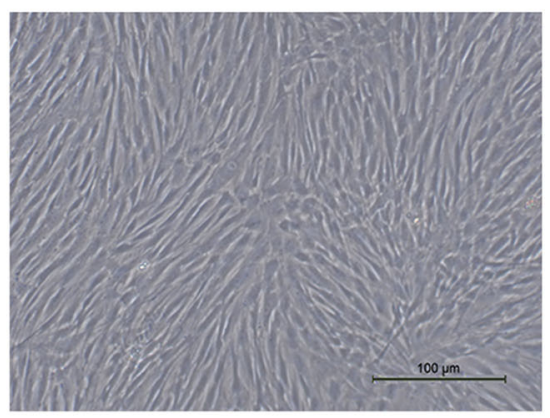

C

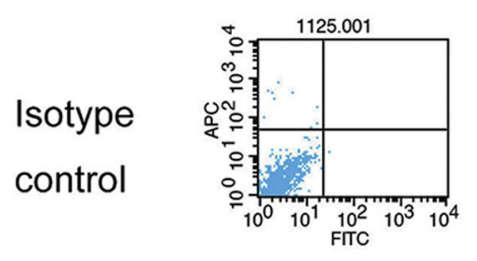

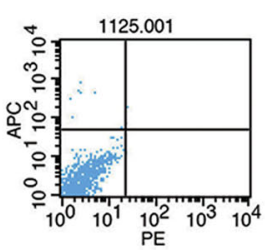
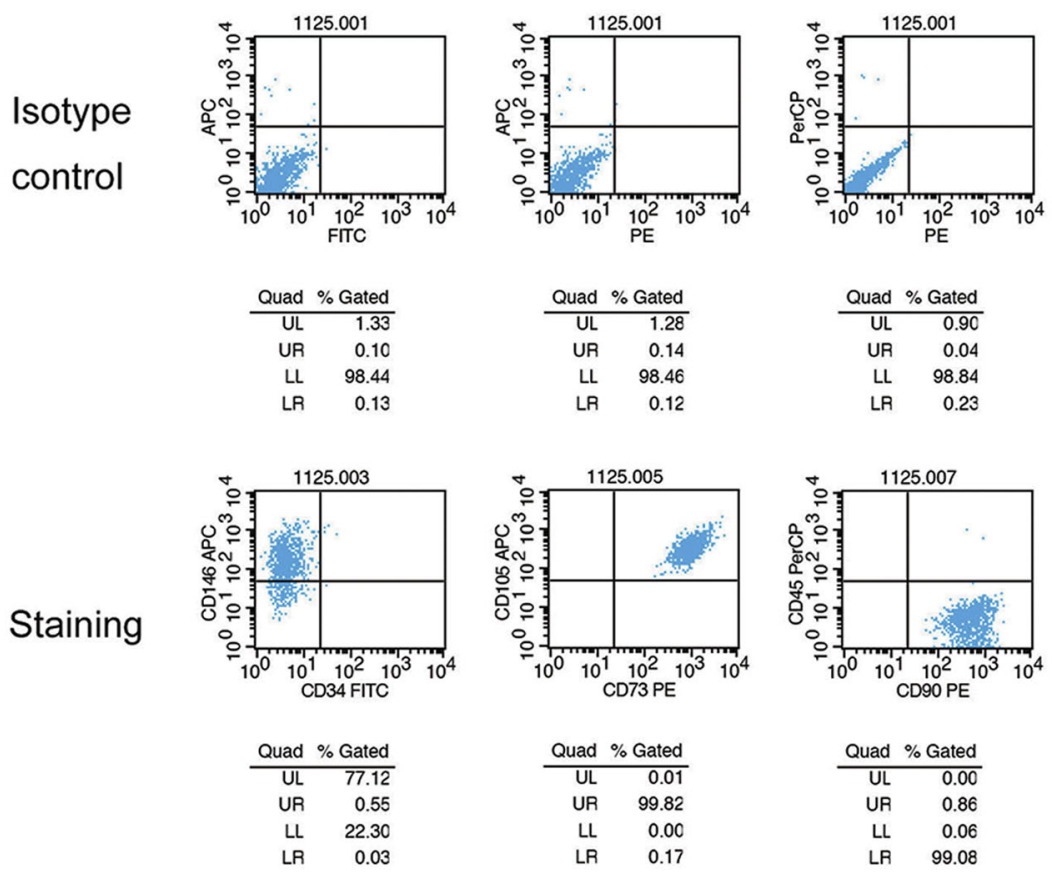

d

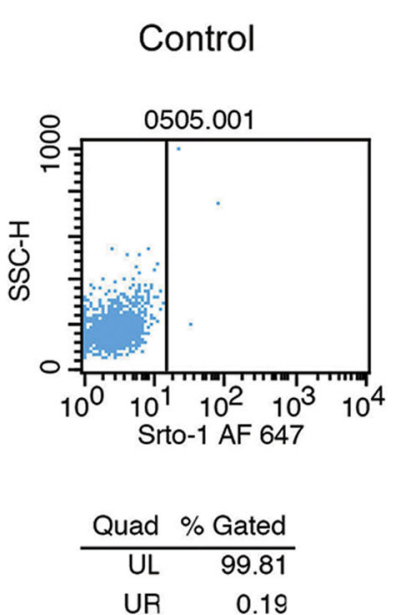

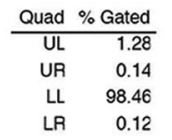
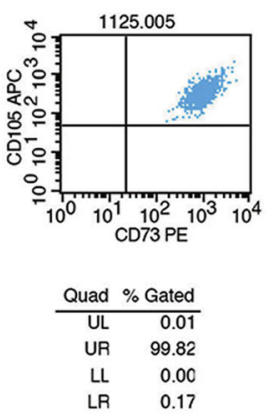
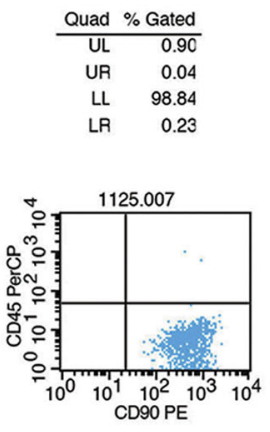

LLL $\quad 98.84$

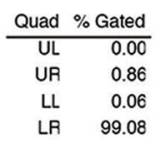

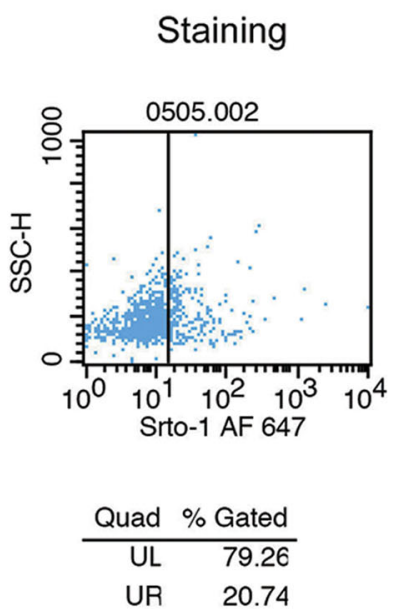

Fig. 1 Isolation and characterization of DPMSCs. $\mathbf{a}$, b Isolated stem cells derived from human dental pulps displayed spindle-like and fibroblastlike morphology. c Flow cytometric analysis revealed that cultured DPMSCs were immunopositive for CD73 (99.99\%), CD90 (99.94\%), CD105 (99.83\%), and CD146 (77.67\%) but immunonegative for CD34 (0.58\%) and CD45 (0.86\%). Mouse lgG isotype control antibodies conjugated to FITC, PE, APC, or PerCP were used as negative controls. d Flow cytometry revealed that cultured DPMSCs were positive for STRO-1 (20.74\%). PBS served as a negative control. Scale bars: $100 \mu \mathrm{m}$ 


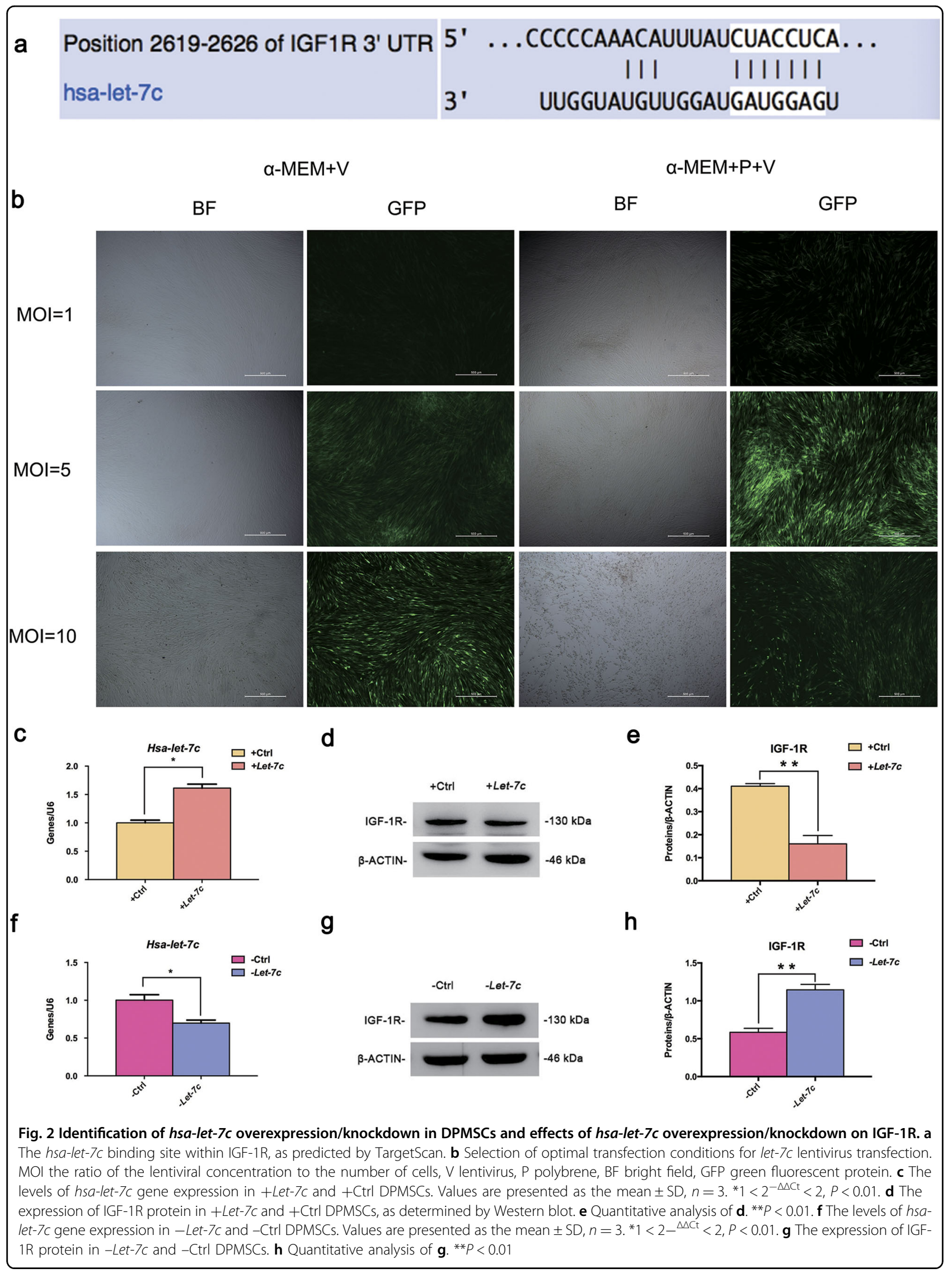




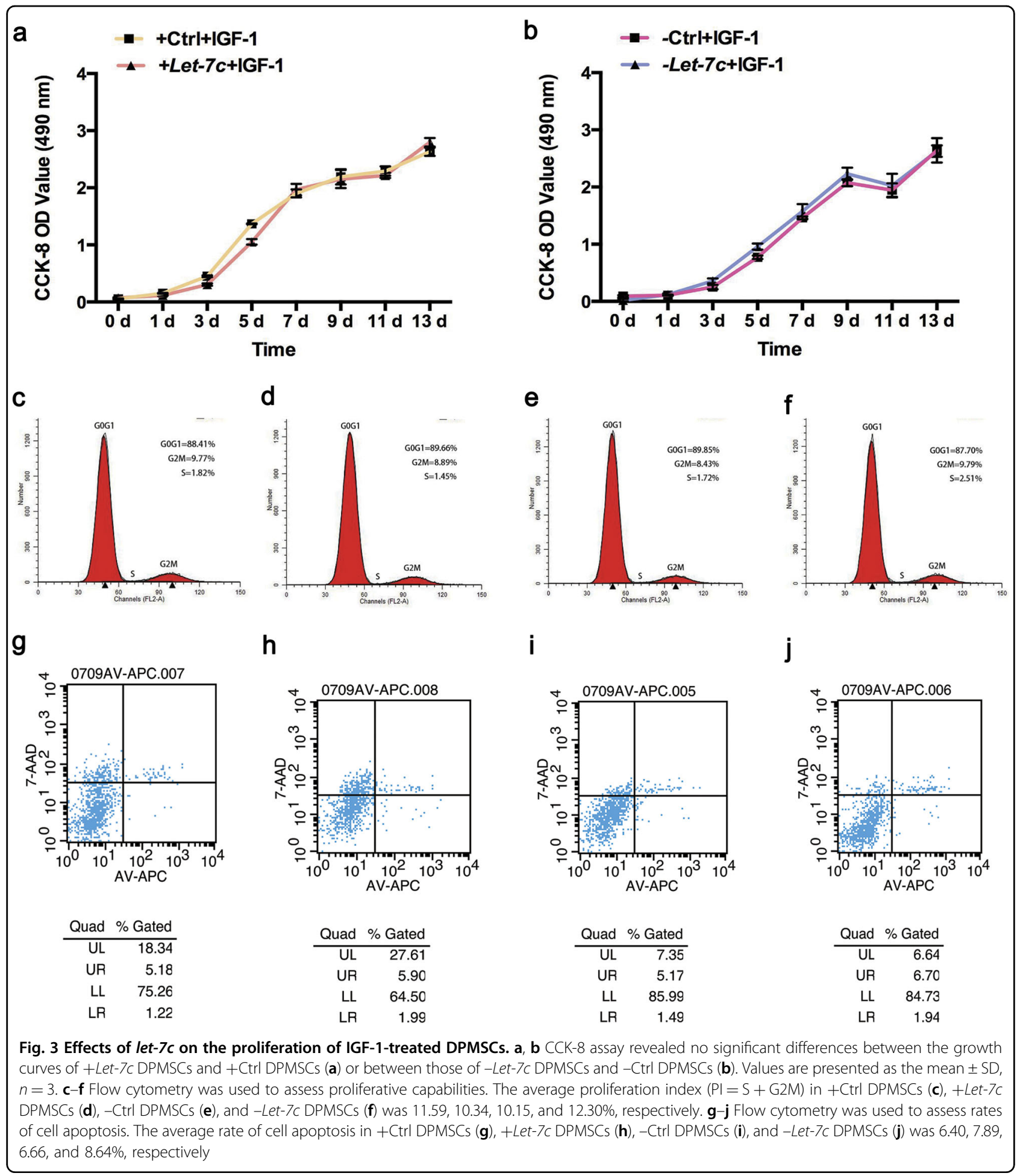

We then determined that IGF-1R was one of the potential gene targets of $h s a-l e t-7 c$ by TargetScan; the putative binding site is shown in Fig. 2a. Let-7c lentiviruses were subsequently designed according to this binding site. To determine the optimal let- $7 c$ lentivirus transfection conditions, let- $7 c$ lentiviruses of different concentrations $(\mathrm{MOI}=1,5,10 ; \mathrm{MOI}$ is the ratio of the lentiviral concentration to the number of cells) were transfected into DPMSCs in either $\alpha$-MEM or $\alpha-M E M+P$ (polybrene). After $72 \mathrm{~h}$ of transfection, cell fate and transfection efficiency were visualized under a fluorescence microscope. The cell morphology of DPMSCs 


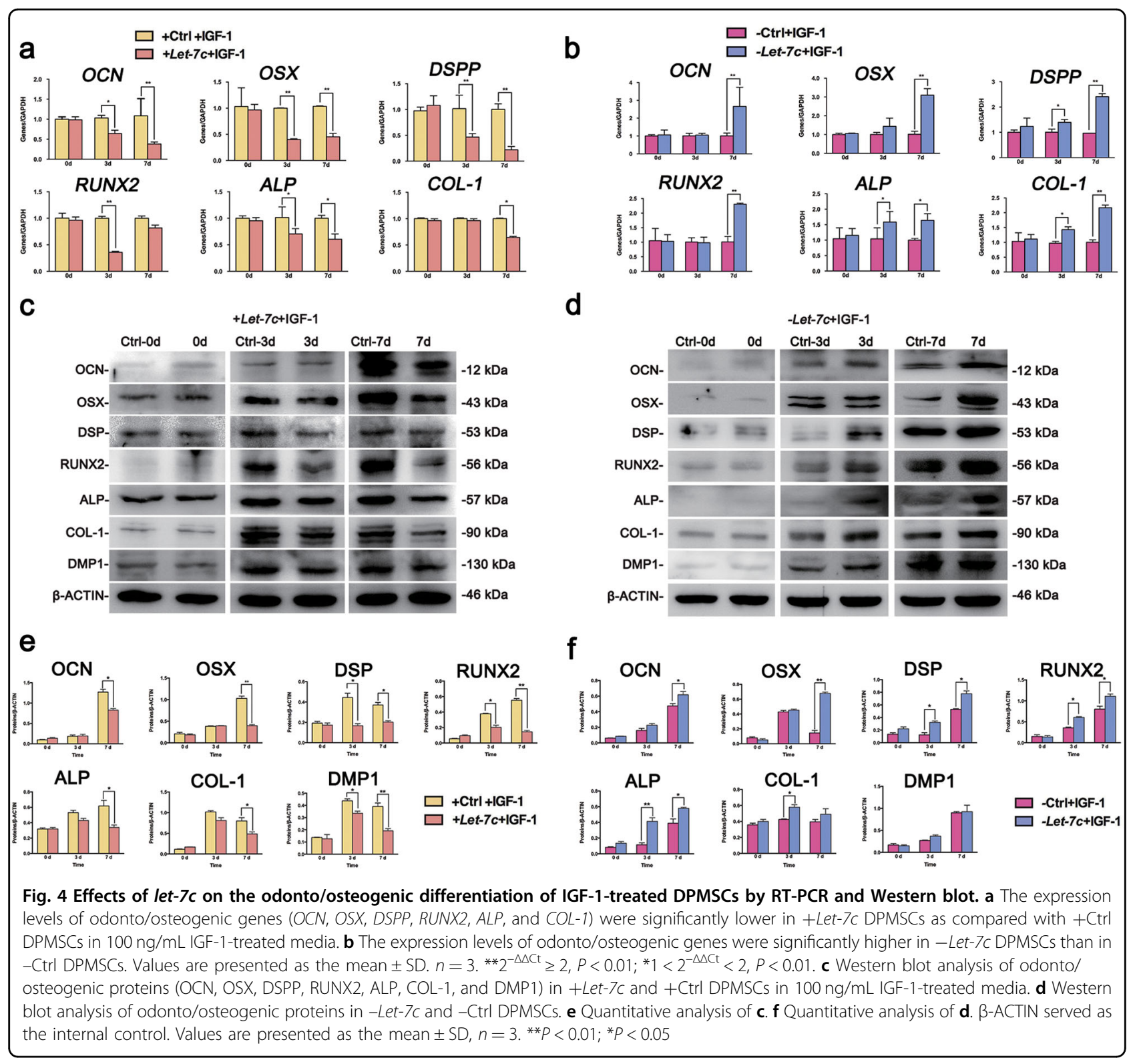

transfected with let-7c lentiviruses at $\mathrm{MOI}=1$ and 5 was normal; however, those transfected at $\mathrm{MOI}=10$ were dysmorphic and had high rates of cell death (Fig. 2b). Moreover, DPMSCs transfected with let- $7 c$ lentiviruses at MOI $=1$ and 10 exhibited fewer GFP-positive cells than those transfected at MOI $=5$ (Fig. 2b). When the MOI was equal to 5, DPMSCs expressed more GFP when transfected in $\alpha-\mathrm{MEM}+\mathrm{P}$ as compared to $\alpha$-MEM only. Under $\mathrm{MOI}=5$ and $\alpha-\mathrm{MEM}+\mathrm{P}$ conditions, DPMSCs presented with the greatest GFP intensity and normal cell morphology. Therefore, this condition was used for subsequent experiments.

To detect whether let-7c levels are up/downregulated in DPMSCs, let-7c expression levels were measured by realtime RT-PCR $6 \mathrm{~h}$ following the transfection of DPMSCs with let-7c lentiviruses. The results showed that expression of the let-7c gene was significantly upregulated in + Let-7c DPMSCs (Fig. 2c), while IGF-1R protein expression was significantly decreased as compared with the + Ctrl group (Fig. 2d, e). Inversely, DPMSCs in the - Let-7c group displayed downregulated let-7c gene expression (Fig. 2f) and increased IGF-1R protein expression as compared with the -Ctrl group (Fig. 2g, h). These data revealed the significant inverse correlation between $h s a-l e t-7 c$ and IGF-1R.

\section{Effects of let-7c on DPMSC proliferation}

The cell counting kit- 8 assay was used to investigate the proliferation of + Let-7c/-Let-7c DPMSCs after treatment with $100 \mathrm{ng} / \mathrm{mL}$ IGF-1 for $0,1,3,5,7,9,11$, and 13 


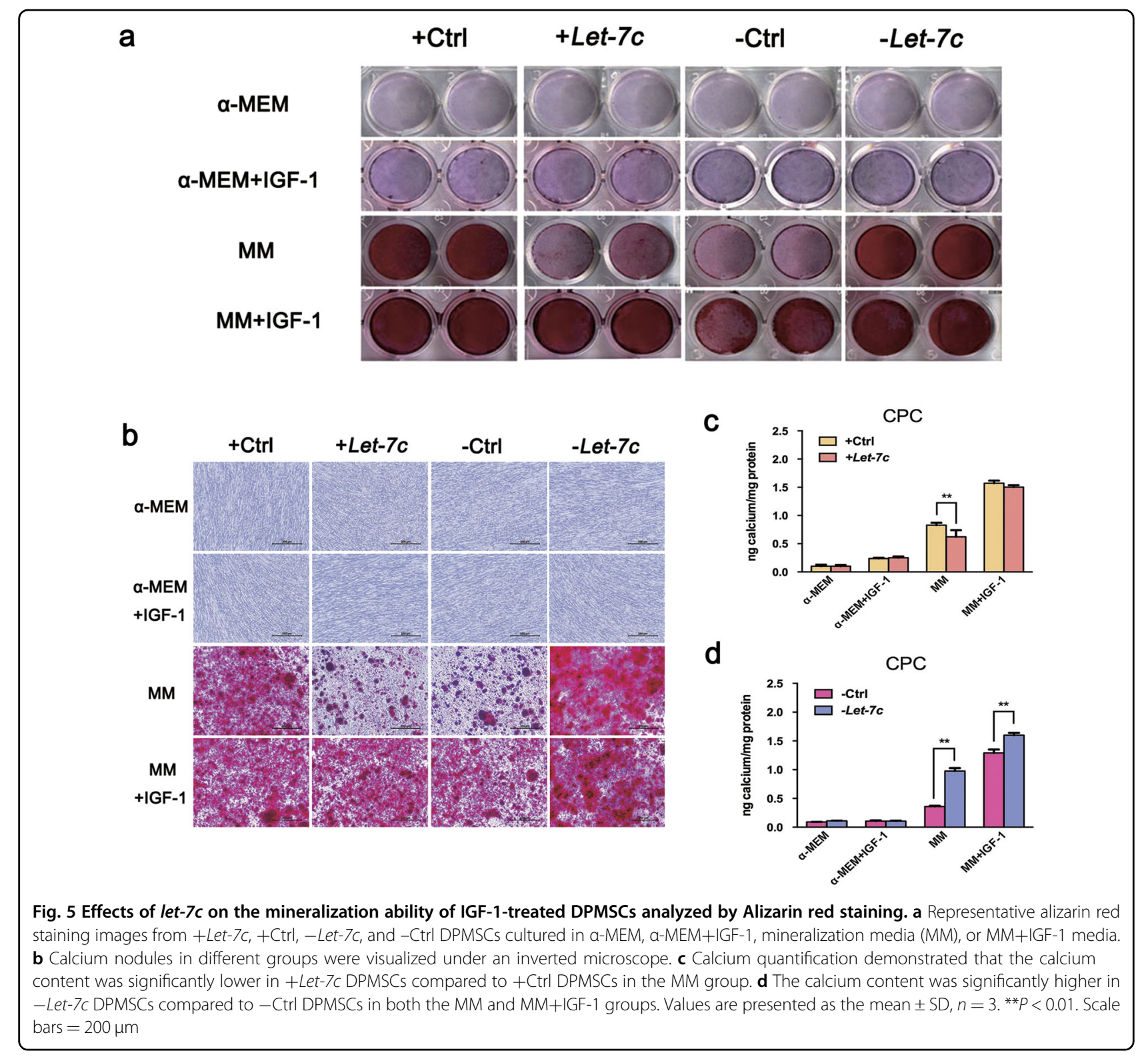

consecutive days. The results demonstrated that the proliferation of DPMSCs in the + Let-7c group was not significantly different from those in the + Ctrl group (Fig. 3a). Similarly, the - Let-7c group presented almost the same proliferation rate as the -Ctrl group between days 0 and 13 (Fig. 3b). FCM revealed that the proliferation index $\left(\mathrm{PI}=\mathrm{S} \%+\mathrm{G}_{2} \mathrm{M} \%\right)$ in the + Let-7c group (11.59\%, Fig. 3d) was very similar to that of the +Ctrl group (10.34\%, Fig. 3c). There was also no significant difference in the proliferation indices of the - Let-7c group (10.15\%, Fig. 3f) and the -Ctrl group (12.3\%, Fig. 3e). Furthermore, FCM demonstrated that the average cell apoptosis rate of DPMSCs in the +Ctrl, + Let- $7 c$, -Ctrl, and - Let-7c groups were $7.89 \%$ (Fig. 3g), 6.40\% (Fig. 3h), 8.64\% (Fig. 3i), and 6.66\% (Fig. 3j), respectively. These data demonstrated that let- $7 c$ had no obvious effects on the proliferative capacity and cell viability of DPMSCs.

\section{Let-7c regulates IGF-1-mediated osteo/odontogenic differentiation of DPMSCs}

To detect the effects of let-7c on the osteo/odontogenic differentiation of DPMSCs, real-time RT-PCR and Western blot were conducted on DPMSCs infected with + Let$7 c /-$ Let- $7 c$ lentiviruses and treated with $100 \mathrm{ng} / \mathrm{mL}$ IGF1. RT-PCR showed that the expression of osteo/odontogenic genes (OCN, OSX, DSPP, RUNX2, ALP, and COL-1) was remarkably decreased in + Let-7c DPMSCs as compared with the + Ctrl group (Fig. 4a). In particular, the expression levels of OCN, OSX, DSPP, and ALP continued to decline between days 3 and 7 (Fig. 4a). The expression 

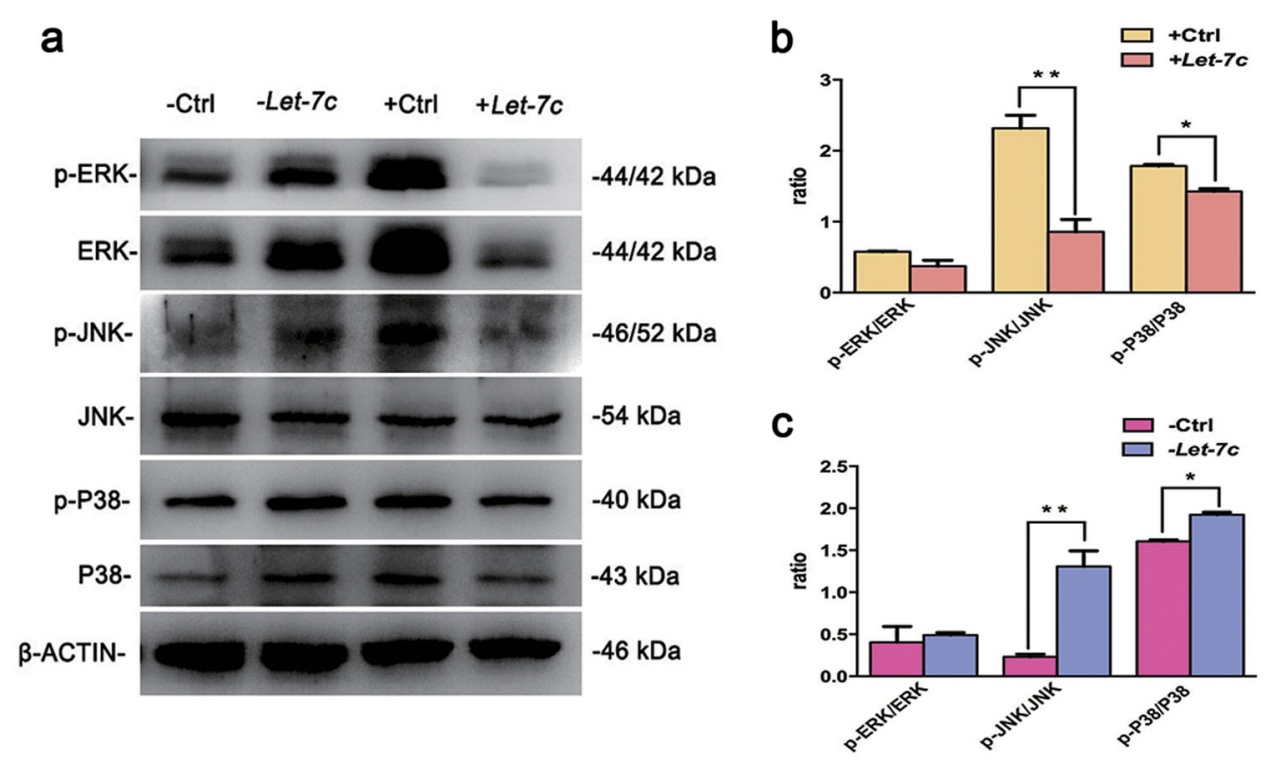

Fig. 6 Involvement of MAPK signaling pathways. a The expression of MAPK-related proteins (ERK, p-ERK, JNK, p-JNK, P38, and p-P38) was analyzed by Western blot in different groups. $\beta$-ACTIN served as the internal control. b Quantitative analysis demonstrated that the p-JNK/JNK and p-P38/P38 ratios were significantly decreased in + Let-7C DPMSCs compared to +Ctrl DPMSCs. c The p-JNKJJNK and p-P38/P38 ratios were significantly increased in -Let-7c DPMSCs compared to -Ctrl DPMSCs. Values are presented as the mean $\pm S D, n=3 .{ }^{* *} P<0.01 ;{ }^{*} P<0.05$

levels of RUNX2 and COL-1 were significantly declining at day 3 and day 7 , respectively (Fig. 4a). Western blot showed that the expression of osteo/odontogenic proteins (OCN, OSX, DSPP, RUNX2, ALP, COL-1, and DMP1) was significantly downregulated from day 3 to day 7 , while there was no significant decrease in the expression of these proteins at day 0 in the + Let-7c group (Fig. 4c, e).

After treatment with $100 \mathrm{ng} / \mathrm{mL}$ IGF-1, the expression levels of osteo/odontogenic genes (OCN, OSX, DSPP, RUNX2, ALP, and COL-1) in the - Let-7c group were obviously upregulated compared to the -Ctrl group (Fig. 4b). In particular, the expression levels of OCN, OSX, and $R U N X 2$ were significantly increased at day 7 in the - Let-7c group as compared to the -Ctrl group (Fig. 4b). The expression levels of $D S P P, A L P$, and $C O L-1$ increased from day 3 to day 7 (Fig. 4b). Western blot showed that the expression of osteo/odontogenic proteins (OCN, OSX, DSPP, RUNX2, ALP, COL-1, and DMP1) was upregulated from day 3 to day 7 , while there was no significant decrease in the expression of these proteins at day 0 in the - Let- $7 c$ group (Fig. $4 \mathrm{~d}, \mathrm{f}$ ).

Alizarin red staining revealed that fewer mineralized nodules were detected in the MM/MM+IGF-1 + Let-7c groups at day 14, as compared with the + Ctrl groups (Fig. 5a, b). There was no significant difference between the + Let- $7 c$ groups and the + Ctrl groups cultured in either $\alpha$-MEM or $\alpha$-MEM+IGF-1 media (Fig. 5a, b). CPC assays further demonstrated that the calcium concentration in the MM-treated + Let- $7 c$ group was significantly lower than that in the + Ctrl group (Fig. 5c).

Notably, the mineralized nodules were much more obvious in the MM/MM+IGF-1 - Let-7c groups as compared with those in the $-\mathrm{Ctrl}$ groups, while no difference was detected between the $\alpha$-MEM/ $\alpha-M E M+I G F-$ $1-$ Let-7c and - Ctrl groups (Fig. 5a, b). CPC assays also revealed that calcium concentrations in the MM/MM +IGF-1 -Let-7c groups were significantly higher than those in the - Ctrl groups (Fig. 5d).

The above findings demonstrated that let-7c plays an important role in the osteo/odontogenic differentiation of DPMSCs.

\section{Effects of let-7c on MAPK pathways in DPMSCs}

To determine the potential involvement of MAPK signaling pathways in + Let-7c/-Let-7c-treated DPMSCs, MAPK-related proteins were detected in DPMSCs following infection with + Let-7c/-Let-7c lentiviruses. Total ERK and p38 protein levels significantly decreased in the + Let- $7 c$ group as compared with the + Ctrl group (Fig. 6a). The levels of phosphorylated ERK, JNK, and p38 were significantly downregulated in the + Let- $7 c$ group in comparison with the + Ctrl group (Fig. 6a). The p-JNK/ JNK and p-p38/p38 intensity ratios were markedly decreased in the + Let- $7 c$ group as compared with those in the + Ctrl group (Fig. $6 \mathrm{~b}$ ). In the - Let- $7 c$ group, the levels of total ERK and p38 as well as phosphorylated ERK, JNK, and p38 were significantly increased compared with the - 


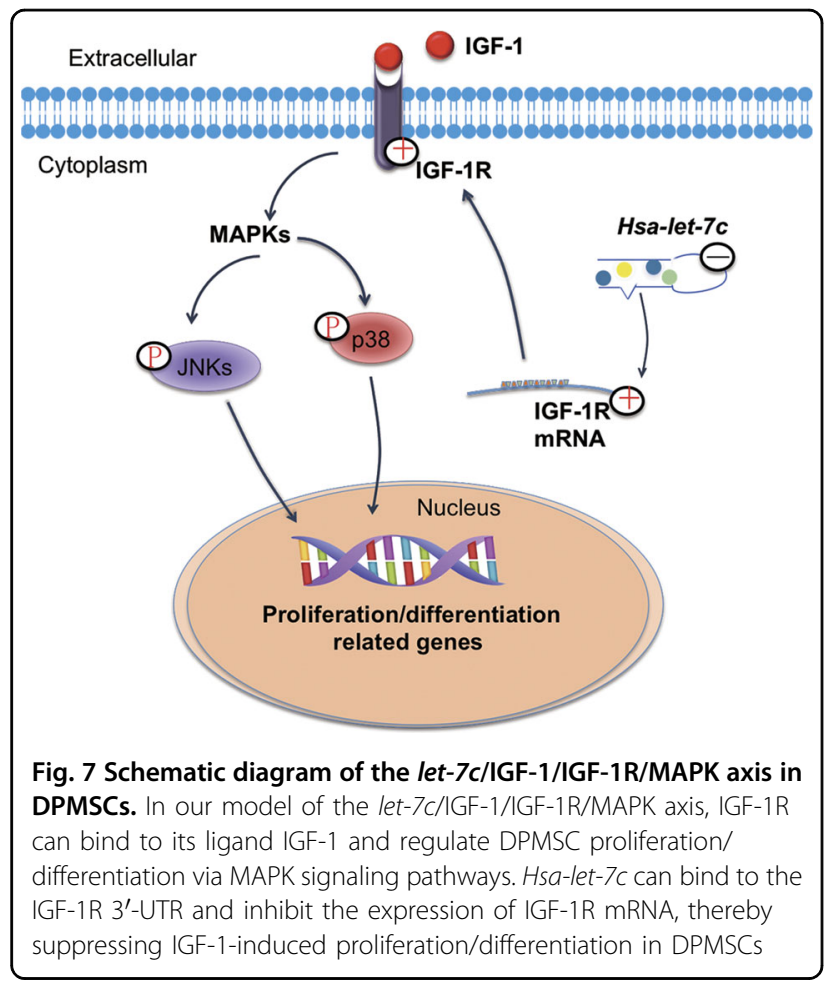

Ctrl group; however, the level of total JNK was decreased (Fig. 6a). The p-JNK/JNK and p-p38/p38 intensity ratios were clearly upregulated in the - Let-7c group compared to the - Ctrl group (Fig. 6c).

\section{Discussion}

MiRNAs act as posttranscriptional regulators of gene expression by targeting the $3^{\prime}$-UTR regions of target mRNAs. In this study, IGF-1R was predicted to be a target of $h s a-l e t-7 c$ by TargetScan and thus we chose to investigate its effects on the biologic activities of IGF-1-treated DPMSCs. In brief, let-7c inversely regulated the expression of IGF-1R protein and suppressed the JNK/P38 MAPK signaling pathways. Furthermore, let-7c had the capacity to inhibit the osteo/odontogenic differentiation of IGF-1-treated DPMSCs. Since MAPK signaling is downstream of IGF-1/IGF-1R $R^{9,33-38}$, we proposed that hsa-let-7c inhibits the odonto/osteogenic differentiation of IGF-1-treated DPMSCs by targeting IGF-1R via the JNK/P38 MAPK pathways (Fig. 7).

In this study, knockdown of let-7c increased the protein expression levels of IGF-1R, while overexpression of let-7c reduced IGF-1R expression, suggesting that IGF-1R is suppressed by let-7c in DPMSCs. Previous studies have shown that IGF-1 and IGF-1R are both targets of the let-7 family and that let-7 is closely associated with the IGF-1/ IGF-1R axis $^{33}$. Let-7 can bring about the upregulation of IGF-1 and IGF-1R, indicating that let-7 may contribute to the upstream regulation of the IGF-1/IGF-1R axis $^{6,9}$. In the presence of IGF-1, expression of let-7 family members usually decreases while BMSCs undergo committed differentiation. Decreases in let-7 may allow for the upregulation of target genes such as IGF-1R, thus modulating the proliferation or differentiation of $\mathrm{MSCs}^{9}$. In short, IGF-1R is a target of $l e t-7 c$, and the latter can suppress the osteo/odontogenic ability of IGF-1-treated DPMSCs by inhibiting the expression of IGF-1R.

The $\mathrm{p}-\mathrm{JNK} / \mathrm{JNK}$ and $\mathrm{p}-\mathrm{p} 38 / \mathrm{p} 38$ intensity ratios distinctly decreased following let-7c overexpression and increased following let-7c knockdown, indicating that the JNK and P38 MAPK pathways are blocked by let-7c in IGF-1-treated DPMSCs. The MAPK and PI3K signaling pathways have been reported to be downstream mechanisms regulated by the IGF-1/IGF-1R axis ${ }^{35}$. siRNA-mediated knockdown of IGF-1 can diminish the activation of ERK1/2 and PI3K. At the same time, either specific inhibitors or knockdown of ERK1/2 and PI3K can effectively abrogate IGF-1/IGF-1R-mediated differentiation $^{33}$. Therefore, the ERK MAPK and PI3K signaling pathways seem to be the downstream mechanisms effecting IGF-1-induced differentiation. Furthermore, P38 MAPK is a downstream target of IGF-1R and IGF-1R may control stem cell quiescence via p38 MAPK signaling ${ }^{34}$. Interestingly, inhibitors of IGF-1R can decrease DPMSC colony-forming units, while inhibitors of p38 can overcome the suppressive effects of IGF-1R inhibition ${ }^{34}$. These findings imply that IGF-1R and p38 MAPK can interact with each other to balance the quiescence and differentiation of DPMSCs. Since $I G F-1 R$ is a target gene of $l e t-7 c$ and is downregulated by let-7c, it seems that decreases in JNK and P38 MAPK signaling are associated with let-7c-mediated suppression of IGF-1R. Furthermore, MAP4K4 is the upstream regulator of the ERK, JNK, and p38 MAPK signaling pathways. MAP4K4 contains a let-7 target site in its $3^{\prime}$-UTR, indicating that let-7 family can regulate MAPK pathways by altering MAP4K4 expression levels ${ }^{39,40}$. Hsa-let-7c-5p can facilitate human enterovirus 71 replication by inhibiting MAP4K4 expression; this mechanism might explain the virus's ability to subvert the JNK pathway ${ }^{41}$. Previous studies have demonstrated that ERK1/2 MAPK negatively regulates let-7 by inducing LIN28 expression through Myc transcription ${ }^{42}$. Meanwhile, others have demonstrated that Ras, an upstream activator of Raf/MAPK, is inhibited by let- 7 family miRNAs ${ }^{43,44}$. Thus, it seems that let-7 and MAPK signaling pathways may function within a positive feedback loop. However, we found no direct evidence that MAPK signaling pathways can be regulated by let- $7 c$ itself. The speculation that JNK and P38 MAPK signaling can be directly regulated by let- $7 c$ still requires more extensive study. Therefore, it is reasonable to propose that the overexpression of let-7c may indirectly downregulate 
the JNK and P38 MAPK signaling pathways by targeting IGF-1R.

ALP, OCN, OSX, RUNX2, COL-1, DSPP/DSP, and DMP-1 are usually recognized as the functional markers of odonto/osteoblast activity, bone/tooth regeneration, and odonto/osteogenesis. Specifically, ALP is an important marker involved in the early stages of osteogenic differentiation and has been widely used to evaluate the osteogenic potential of cells in vitro ${ }^{45,46}$. OCN is a latestage marker of osteogenic differentiation and mainly appears in mature bone tissues ${ }^{47}$. The transcription factor RUNX2 serves as an early transcriptional regulator of osteogenic differentiation and its downstream target OSX is involved in both early and late stages of osteogenic differentiation ${ }^{48,49}$. COL- 1 is the most abundant protein in bone and is the main component of the bone matrix. Higher COL-1 expression may reflect matrix mineralization in mineralized bones ${ }^{50}$. DSPP and DSP are odontoblast-specific markers, which are highly expressed in dentin and predentin structures and play critical roles in dentinogenesis ${ }^{51}$. DMP-1 is an extracellular matrix protein mainly expressed in odontoblasts and is essential for dentin mineralization ${ }^{52}$. DSPP may act as a downstream effector molecule of DMP1 during dentinogenesis. At days 3 and 7 following the overexpression of let-7c in $100 \mathrm{ng} / \mathrm{mL}$ IGF-1-treated DPMSCs, the mineralization capacity and expression of osteo/odontogenic markers (ALP, OCN, OSX, RUNX2, COL-1, and DMP1) were distinctly downregulated. Inversely, the mineralization capacity and expression of osteo/odontogenic markers were clearly upregulated at days 3 and 7 in IGF-1-treated - let-7c DPMSCs. These results revealed that let-7c could suppress the IGF-1-induced osteo/odontogenic differentiation of DPMSCs. Together, it is reasonable to suggest that let-7c suppresses the IGF-1-induced osteo/odontogenic differentiation of DPMSCs by inhibiting the expression of IGF-1R.

The IGF-1/IGF-1R axis directly participates in the regulation of gene expression and osteoblast differentiation, a critical step in the maintenance of bone homeostasis ${ }^{53,54}$. IGF-1R possesses the ability to translocate to the cell nucleus and elicit genomic activities triggered by transcription factors. Nuclear IGF-1R has functional activities similar to transcription factors, providing an additional layer of biological regulation at a genome-wide level ${ }^{55}$. By stimulating the action of IGF1R, IGF-1 acts as an important mitogenic factor to promote cell proliferation and differentiation ${ }^{56}$. Our previous studies have demonstrated that exogenous IGF1 can modify cellular morphology and ultrastructure as well as promote the proliferation and differentiation of DPMSCs, hSCAPs, and hPDLSCs ${ }^{13-15}$. Exogenous IGF-1 can enhance the proliferative and osteo/odontogenic abilities of DPMSCs by activating the ERK and p38 MAPK signaling pathways ${ }^{13}$. In addition, IGF-1 can induce the osteogenic differentiation of hPDLSCs via MAPK signaling ${ }^{15}$. In this study, let-7c significantly downregulated both JNK/P38 MAPK signaling and the expression of the upstream regulator IGF-1R and inhibited the committed differentiation of IGF-1-treated DPMSCs. In short, let-7c expression depressed IGF-1R expression, which subsequently downregulated the JNK/ P38 MAPK signaling pathway and finally led to the downregulation of osteo/odontogenic differentiation in DPMSCs.

In this study, overexpression of miRNA let-7c led to the downregulation of IGF-1R and the downstream JNK/P38 MAPK signaling pathways, subsequently resulting in the weakened osteo/odontogenic differentiation of IGF-1treated DPMSCs. Inversely, deletion of let-7c caused upregulation of IGF-1R and its downstream JNK/P38 MAPK signaling, resulting in the enhanced osteo/odontogenic differentiation of IGF-1-treated DPMSCs. Thus, it is speculated that a let-7c/IGF-1/IGF-1R/MAPK axis may exist to effect let-7c-mediated changes in IGF-1-treated DPMSCs. Hsa-let-7c inhibited the osteo/odontogenic differentiation of IGF-1-treated DPMSCs by suppressing the expression of IGF-1R on the membrane. This decrease in IGF-1R expression then brought about the inhibition of the cytoplasmic JNK/P38 MAPK signaling pathways. Decreases in the amount of phosphorylated JNK/P38 in the nucleus subsequently resulted in the downregulation of downstream genes associated with the committed differentiation of DPMSCs (Fig. 7). Taken together, the modification of osteo/odontogenic differentiation of IGF1-treated DPMSCs by let-7c was mediated by IGF-1/IGF$1 \mathrm{R}$ and the downstream JNK/P38 MAPK signaling pathways. Further study is required to explore the potential applications of this axis in tooth engineering as well as bone reconstruction.

\footnotetext{
Acknowledgements

This work was supported by the National Natural Science Foundation of China (No. 81371144), the Medical Talent Project of Jiangsu Province (ZDRCA2016086), the Science and Technology Development Project of Jiangsu Province (BE2017731), and the Priority Academic Program Development of Jiangsu Higher Education Institutions (PAPD, No. 2014-37).
}

\footnotetext{
Author details

${ }^{1}$ Endodontic Department, Hefei Hospital of Stomatology, 265 Changjiang Middle Road, 230001 Hefei, Anhui, China. ${ }^{2}$ Key Laboratory of Oral Diseases of Jiangsu Province and Stomatological Institute of Nanjing Medical University, 136 Hanzhong Road, 210029 Nanjing, Jiangsu, China. ${ }^{3}$ Guiyang Hospital of Stomatology, 18 Xingguan Road, 550002 Guiyang, Guizhou, China.

${ }^{4}$ Department of Stomatology, Nanjing Integrated Traditional Chinese and Western Medicine Hospital Affiliated with Nanjing University of Chinese Medicine, 210014 Nanjing, Jiangsu, China. ${ }^{5}$ Endodontic Department, School of Stomatology, Nanjing Medical University, 136 Hanzhong Road, 210029 Nanjing, Jiangsu, China
} 


\section{Authors' contributions}

G.X.L. and S.M.: project conception and design, data collection, and/or assembly, data analysis and interpretation, manuscript drafting; Y.L.: RT-PCR, cell culture; Y.Y.: manuscript drafting, technical support; Y.X.Z:: Western blot analysis, alizarin red staining; Y.L.: FCM, statistical support; L.J.: CCK-8 assay, cell identification; Z.W.: technical support, lentivirus ordering; J.Y.: study design, technical support, and final approval of manuscript.

\section{Conflict of interest}

The authors declare that they have no conflict of interest.

\section{Publisher's note}

Springer Nature remains neutral with regard to jurisdictional claims in published maps and institutional affiliations.

Received: 10 September 2017 Revised: 8 December 2017 Accepted: 25 December 2017.

Published online: 13 April 2018

\section{References}

1. Ishaq, R. A., Soliman, S. A., Foda, M. Y. \& Fayed, M. M. Insulin-like growth factor I: a biologic maturation indicator. Am. J. Orthod. Dentofac. Orthop. 142, 654-661 (2012).

2. Xian, L. et al. Matrix IGF-1 maintains bone mass by activation of mTOR in mesenchymal stem cells. Nat. Med. 18, 1095-1101 (2012).

3. Crane, J. L. et al. IGF-1 signaling is essential for differentiation of mesenchymal stem cells for peak bone mass. Bone Res. 1, 186-194 (2013).

4. Zhang, J. et al. SUMOylation of insulin-like growth factor 1 receptor, promotes proliferation in acute myeloid leukemia. Cancer Lett. 357, 297-306 (2015).

5. Lewis, D. A., Travers, J. B., Somani, A. K. \& Spandau, D. F. The IGF-1/IGF-1R signaling axis in the skin: a new role for the dermis in aging-associated skin cancer. Oncogene 29, 1475-1485 (2010).

6. Ma, S. et al. IGF-1/IGF-1R/hsa-let-7c axis regulates the committed differentiation of stem cells from apical papilla. Sci. Rep. 6, 36922 (2016).

7. Mahran, Y. F. et al. Growth hormone ameliorates the radiotherapy-induced ovarian follicular loss in rats: impact on oxidative stress, apoptosis and IGF-1/ IGF-1R axis. PLOS ONE 10, e0140055 (2015).

8. Saikali, Z., Setya, H., Singh, G. \& Persad, S. Role of IGF-1/IGF-1R in regulation of invasion in DU145 prostate cancer cells. Cancer Cell Int. 8, 10 (2008).

9. Huat, T. J., Khan, A. A., Abdullah, J. M., Idris, F. M. \& Jaafar, H. MicroRNA expression profile of neural progenitor-like cells derived from rat bone marrow mesenchymal stem cells under the influence of IGF-1, bFGF and EGF. Int. J. Mol. Sci. 16, 9693-9718 (2015).

10. Mellough, C. B. et al. IGF-1 signaling plays an important role in the formation of three-dimensional laminated neural retina and other ocular structures from human embryonic stem cells. Stem Cells 33, 2416-2430 (2015).

11. Ohlsson, C. et al. Older men with low serum IGF-1 have an increased risk of incident fractures: the MrOS Sweden study. J. Bone Miner. Res. 26, 865-872 (2011).

12. Nguyen, T. T., Sheppard, A. M., Kaye, P. L. \& Noakes, P. G. IGF-I and insulin activate mitogen-activated protein kinase via the type 1 IGF receptor in mouse embryonic stem cells. Reproduction 134, 41-49 (2007).

13. LV, T. et al. Insulin-like growth factor 1 promotes the proliferation and committed differentiation of human dental pulp stem cells through MAPK pathways. Arch. Oral Biol. 72, 116-123 (2016).

14. Wang, S. et al. Insulin-like growth factor 1 can promote the osteogenic differentiation and osteogenesis of stem cells from apical papilla. Stem Cell Res. 8 346-356 (2012)

15. $Y u, Y$. et al. Insulin-like growth factor 1 enhances the proliferation and osteogenic differentiation of human periodontal ligament stem cells via ERK and JNK MAPK pathways. Histochem. Cell Biol. 137, 513-525 (2012).

16. Mukherjee, A. \& Rotwein, P. Akt promotes BMP2-mediated osteoblast differentiation and bone development. J. Cell Sci. 122, 716-726 (2009).

17. Bartel, D. P. microRNAs: genomics, biogenesis, mechanism, and function. Cell 116, 281-297 (2004)

18. Serpico, D., Molino, L. \& Di Cosimo, S. microRNAs in breast cancer development and treatment. Cancer Treat. Rev. 40, 595-604 (2014).
19. Chien, C. S. et al. Lin28B/Let-7 regulates expression of Oct4 and Sox2 and reprograms oral squamous cell carcinoma cells to a stem-like state. Cancer Res. 75, 2553-2565 (2015).

20. Kong, D. et al. Loss of let-7 up-regulates EZH2 in prostate cancer consistent with the acquisition of cancer stem cell signatures that are attenuated by BRDIM. PLOS ONE 7, e33729 (2012).

21. Liu, C. et al. Distinct microRNA expression profiles in prostate cancer stem/ progenitor cells and tumor-suppressive functions of let-7. Cancer Res. 72, 3393-3404 (2012).

22. Yamada, H. et al. Detailed characterization of a homozygously deleted region corresponding to a candidate tumor suppressor locus at 21q11-21 in human lung cancer. Genes Chromosomes Cancer 47, 810-818 (2008).

23. Boyerinas, B., Park, S. M., Hau, A., Murmann, A. E. \& Peter, M. E. The role of let-7 in cell differentiation and cancer. Endocr. Relat. Cancer 17, F19-F36 (2010).

24. Wei, J. et al. let-7 enhances osteogenesis and bone formation while repressing adipogenesis of human stromal/mesenchymal stem cells by regulating HMGA2. Stem Cells Dev. 23, 1452-1463 (2014).

25. Crobu, F. et al. Differentiation of single cell derived human mesenchymal stem cells into cells with a neuronal phenotype: RNA and microRNA expression profile. Mol. Biol. Rep. 39, 3995-4007 (2012).

26. Sekar, D. et al. Role of microRNA 21 in mesenchymal stem cell (MSC) differentiation: a powerful biomarker in MSCs derived cells. Curr. Pharm. Biotechnol. 16, 43-48 (2015).

27. Weber, M., Sotoca, A. M., Kupfer, P., Guthke, R. \& van Zoelen, E. J. Dynamic modelling of microRNA regulation during mesenchymal stem cell differentiation. BMC Syst. Biol. 7, 124 (2013).

28. Gronthos, S., Mankani, M., Brahim, J., Robey, P. G. \& Shi, S. Postnatal human dental pulp stem cells (DPSCs) in vitro and in vivo. Proc. Natl Acad. Sci. USA 97, 13625-13630 (2000)

29. Gronthos, S. et al. Stem cell properties of human dental pulp stem cells. J. Dent. Res. 81, 531-535 (2002).

30. Yu, J. et al. Differentiation of dental pulp stem cells into regular-shaped dentinpulp complex induced by tooth germ cell conditioned medium. Tissue Eng. 12, 3097-3105 (2006)

31. Aonuma, $\mathrm{H}$. et al. Characteristics and osteogenic differentiation of stem/progenitor cells in the human dental follicle analyzed by gene expression profiling. Cell Tissue Res. 350, 317-331 (2012).

32. Bustin, S. A. Absolute quantification of mRNA using real-time reverse transcription polymerase chain reaction assays. J. Mol. Endocrinol. 25, 169-193 (2000).

33. Shen, G. et al. Upstream and downstream mechanisms for the promoting effects of IGF-1 on differentiation of spermatogonia to primary spermatocytes. Life Sci. 101, 49-55 (2014).

34. Vandomme, J. et al. Insulin-like growth factor 1 receptor and p38 mitogenactivated protein kinase signals inversely regulate signal transducer and activator of transcription 3 activity to control human dental pulp stem cell quiescence, propagation, and differentiation. Stem Cells Dev. 23, 839-851 (2014).

35. Koike, $M$. et al. Insulin-like growth factor-1 rescues the mutated FGF receptor 3 (G380R) expressing ATDC5 cells from apoptosis through phosphatidylinositol 3-kinase and MAPK. J. Bone Miner. Res. 18, 2043-2051 (2003).

36. Allen, T. R., Krueger, K. D., Hunter, W. J. 3rd \& Agrawal, D. K. Evidence that insulin-like growth factor- 1 requires protein kinase C-epsilon, PI3-kinase and mitogen-activated protein kinase pathways to protect human vascular smooth muscle cells from apoptosis. Immunol. Cell Biol. 83, 651-667 (2005).

37. Parrizas, M. Saltiel, A. R. \& LeRoith, D. Insulin-like growth factor 1 inhibits apoptosis using the phosphatidylinositol 3'-kinase and mitogen-activated protein kinase pathways. J. Biol. Chem. 272, 154-161 (1997).

38. Remacle-Bonnet, M. M. et al. Insulin-like growth factor-l protects colon cancer cells from death factor-induced apoptosis by potentiating tumor necrosis factor alpha-induced mitogen-activated protein kinase and nuclear factor kappaB signaling pathways. Cancer Res. 60, 2007-2017 (2000).

39. Tan, $X$. et al. Cellular microRNA Let-7a suppresses KSHV replication through targeting MAP4K4 signaling pathways. PLOS ONE 10, e0132148 (2015)

40. Liu, A. W. et al. ShRNA-targeted MAP4K4 inhibits hepatocellular carcinoma growth. Clin. Cancer Res. 17, 710-720 (2011).

41. Zhou, B et al. Hsa-let-7c-5p augments enterovirus 71 replication through viral subversion of cell signaling in rhabdomyosarcoma cells. Cell Biosci. 7, 7 (2017).

42. Dangi-Garimella, S. et al. Raf kinase inhibitory protein suppresses a metastasis signalling cascade involving LIN28 and let-7. EMBO J. 28, 347-358 (2009).

43. Choudhury, S. N. \& Li, Y. miR-21 and let-7 in the Ras and NF-kappaB pathways. MicroRNA 1, 65-69 (2012). 
44. Johnson, S. M. et al. RAS is regulated by the let-7 microRNA family. Cell 120, 635-647 (2005).

45. Hong, D. et al. Morphological and proteomic analysis of early stage of osteoblast differentiation in osteoblastic progenitor cells. Exp. Cell Res. 316, 2291-2300 (2010).

46. Bakopoulou, A. et al. Comparative analysis of in vitro osteo/odontogenic differentiation potential of human dental pulp stem cells (DPSCs) and stem cells from the apical papilla (SCAP). Arch. Oral Biol. 56, 709-721 (2011).

47. Bai, Y. et al. Formation of bone-like tissue by dental follicle cells co-cultured with dental papilla cells. Cell Tissue Res. 342, 221-231 (2010).

48. Baek, W. Y. et al. Positive regulation of adult bone formation by osteoblastspecific transcription factor osterix. J. Bone Miner. Res. 24, 1055-1065 (2009).

49. Komori, T. Regulation of osteoblast differentiation by Runx2. Adv. Exp. Med. Biol. 658, 43-49 (2010)

50. Zhong, W. J. et al. Periostin-like-factor-induced bone formation within orthopedic maxillary expansion. Orthod. Craniofac. Res. 14, 198-205 (2011).

51. Suzuki, S., Haruyama, N., Nishimura, F. \& Kulkarni, A. B. Dentin sialophosphoprotein and dentin matrix protein-1: two highly phosphorylated proteins in mineralized tissues. Arch. Oral Biol. 57, 1165-1175 (2012).
52. Gibson, M. P. et al. The rescue of dentin matrix protein 1 (DMP1)-deficient tooth defects by the transgenic expression of dentin sialophosphoprotein (DSPP) indicates that DSPP is a downstream effector molecule of DMP1 in dentinogenesis. J. Biol. Chem. 288, 7204-7214 (2013).

53. Darvin, P., Joung, Y. H. \& Yang, Y. M. JAK2-STAT5B pathway and osteoblast differentiation. JAK-STAT 2, e24931 (2013).

54. Joung, Y. H. et al. MSM enhances GH signaling via the Jak2/STAT5b pathway in osteoblast-like cells and osteoblast differentiation through the activation of STAT5b in MSCS. PLOS ONE 7, e47477 (2012).

55. Sarfstein, R. \& Werner, H. Minireview: nuclear insulin and insulin-like growth factor-1 receptors: a novel paradigm in signal transduction. Endocrinology 154, 1672-1679 (2013).

56. Mairet-Coello, G., Tury, A. \& DiCicco-Bloom, E. Insulin-like growth factor-1 promotes $\mathrm{G}(1) / \mathrm{S}$ cell cycle progression through bidirectional regulation of cyclins and cyclin-dependent kinase inhibitors via the phosphatidylinositol 3kinase/Akt pathway in developing rat cerebral cortex. J. Neurosci. 29, 775-788 (2009). 